\title{
In vivo measurements document the dynamic cellular kinetics of chronic lymphocytic leukemia B cells
}

Bradley T. Messmer, ${ }^{1}$ Davorka Messmer, ${ }^{1}$ Steven L. Allen, ${ }^{1,2,3}$ Jonathan E. Kolitz,, Prasad Kudalkar, ${ }^{2,3}$ Denise Cesar, ${ }^{4}$ Elizabeth J. Murphy, ${ }^{5}$ Prasad Koduru, ${ }^{6,7}$ Manlio Ferrarini, ${ }^{8}$ Simona Zupo, ${ }^{8}$ Giovanna Cutrona, ${ }^{8}$ Rajendra N. Damle, $, 1,2,3$ Tarun Wasil, ${ }^{9,10}$ Kanti R. Rai, ${ }^{1,9,10}$ Marc K. Hellerstein, ${ }^{4,11}$ and Nicholas Chiorazzi ${ }^{1,2,3}$

${ }^{1}$ Institute for Medical Research, North Shore-LIJ Research Institute, Manhasset, New York, USA. ${ }^{2}$ Department of Medicine, North Shore University Hospital, Manhasset, New York, USA. ${ }^{3}$ Department of Medicine, New York University School of Medicine, New York, New York, USA. ${ }^{4}$ Department of Nutritional Sciences, University of California, Berkeley, California, USA. ${ }^{5}$ KineMed Inc., Emeryville, California, USA. ${ }^{6}$ Department of Laboratories, North Shore University Hospital, Manhasset, New York, USA. ${ }^{7}$ Department of Pathology, New York University School of Medicine, New York, New York, USA. ${ }^{8}$ Division of Medical Oncology C, Istituto Nazionale per la Ricerca sul Cancro, Genova, and Dipartimento di Oncologia Clinica e Sperimentale, Universitá di Genova, Genova, Italy. ${ }^{9}$ Department of Medicine, Long Island Jewish Medical Center, New Hyde Park, New York, USA. ${ }^{10}$ Department of Medicine, Albert Einstein College of Medicine, Bronx, New York, USA. ${ }^{11}$ Department of Medicine, University of California and San Francisco General Hospital, San Francisco, California, USA.

Due to its relatively slow clinical progression, B cell chronic lymphocytic leukemia (B-CLL) is classically described as a disease of accumulation rather than proliferation. However, evidence for various forms of clonal evolution suggests that B-CLL clones may be more dynamic than previously assumed. We used a nonradioactive, stable isotopic labeling method to measure B-CLL cell kinetics in vivo. Nineteen patients drank an aliquot of deuterated water $\left({ }^{2} \mathrm{H}_{2} \mathrm{O}\right)$ daily for 84 days, and ${ }^{2} \mathrm{H}$ incorporation into the deoxyribose moiety of DNA of newly divided B-CLL cells was measured by gas chromatography/mass spectrometry, during and after the labeling period. Birth rates were calculated from the kinetic profiles. Death rates were defined as the difference between calculated birth and growth rates. These analyses demonstrated that the leukemic cells of each patient had definable and often substantial birth rates, varying from $0.1 \%$ to greater than $1.0 \%$ of the entire clone per day. Those patients with birth rates greater than $0.35 \%$ per day were much more likely to exhibit active or to develop progressive disease than those with lower birth rates Thus, B-CLL is not a static disease that results simply from accumulation of long-lived lymphocytes. Rather, it is a dynamic process composed also of cells that proliferate and die, often at appreciable levels. The extent to which this turnover occurs has not been previously appreciated. A correlation between birth rates and disease activity and progression appears to exist, which may help identify patients at risk for worsening disease in advance of clinical deterioration.

\section{Introduction}

B cell chronic lymphocytic leukemia (B-CLL) is characterized by a lymphocytosis of mature-appearing clonal $\mathrm{CD}^{+} \mathrm{B}$ lymphocytes (1). B-CLL cells arise from the bone marrow and infiltrate lymphoid tissues such as lymph nodes and the spleen. All of these are sites of leukemic cell proliferation $(2,3)$, as very few dividing cells are observed in the blood (4). The prevailing view of B-CLL is that it is an accumulative disease (5). However, the inability to precisely define an underlying defect in apoptosis in the leukemic clone (6) and evidence supporting clonal evolution (7) suggest that this view may be too simplistic.

The clinical course of B-CLL patients varies widely. Patients can have stable disease, in terms of leukemic cell load and clinical symptoms, for a decade or longer (8). Several molecular corre-

Nonstandard abbreviations used: B-CLL, B cell chronic lymphocytic leukemia; ECIB, extracorporeal irradiation of blood; GC/MS, gas chromatography/mass spectrometry; ${ }^{2} \mathrm{H}_{2} \mathrm{O}$, deuterated "heavy" water; PMNL, polymorphonuclear leukocyte; WBC, white blood cell; ZAP-70, $\zeta$ chain-associated protein $70 \mathrm{kDa}$.

Conflict of interest: M. Hellerstein, in conjunction with the University of California, holds a patent on the use of the deuterium labeling technique used to determine the kinetics of cell replication in vivo. This patent is currently licensed to KineMed Inc., a for-profit company of which M. Hellerstein is a cofounder and shareholder. E. Murphy is an employee of KineMed Inc.

Citation for this article: $J$. Clin. Invest. 115:755-764 (2005). doi:10.1172/JCI200523409. lates of poor clinical outcome have emerged in recent years. The absence of mutations in the Ig variable region genes of B-CLL clones has significant prognostic value, correlating with a poor outcome $(9,10)$. Expression of $\xi$ chain-associated protein 70 $\mathrm{kDa}(\mathrm{ZAP}-70) \mathrm{mRNA}$ or protein $(11,12)$ and the presence of $\mathrm{CD} 38$ on the leukemic cell surface also presage poor outcome $(9,13-16)$. Several cytogenetic abnormalities (in particular, deletions at $17 \mathrm{p} 13$ and $11 \mathrm{q} 22-23$ ) are associated with unfavorable clinical courses, whereas a deletion at 13q14, in the absence of other abnormalities, bodes a slightly better clinical outcome (17). Finally, the doubling time of circulating B-CLL cells has prognostic value $(18,19)$ that may be applied in a clinically useful proliferation index $(20,21)$.

The kinetics at which B-CLL cells are produced, are anatomically exchanged, and die has been an area of interest for several decades (22). A variety of approaches, including infusion of chromium-labeled leukemic cells (23), in vivo labeling with thymidine and other nucleic acid precursors (24), and the extracorporeal irradiation of blood (ECIB) (25), have been used to address these issues. However, these early studies were limited by small patient numbers, short labeling and observation times, limited computing power to fit models, and difficulty in obtaining highly enriched leukemic cells. There has not been a kinetic study using modern technologies informed by current molecular markers. 
Table 1

Clinical and laboratory features of the B-CLL patients enrolled in this study

\begin{tabular}{|c|c|c|c|c|c|c|c|c|c|c|c|c|c|c|}
\hline \multirow[t]{2}{*}{ Patient } & \multirow[t]{2}{*}{ Age } & \multirow[t]{2}{*}{ Sex } & \multirow[t]{2}{*}{$\begin{array}{l}\text { Rai } \\
\text { stage }\end{array}$} & \multirow{2}{*}{$\begin{array}{c}\text { Years } \\
\text { since } \\
\text { diagnosis }\end{array}$} & \multirow{2}{*}{$\begin{array}{l}\text { Active or } \\
\text { progressive } \\
\text { disease }\end{array}$} & \multirow{2}{*}{$\begin{array}{l}\text { Treatment } \\
\text { e (years) }\end{array}$} & \multirow{2}{*}{ 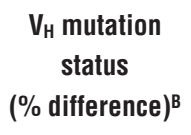 } & \multirow[t]{2}{*}{$\begin{array}{c}\text { CD38 } \\
\text { (\% cells) }\end{array}$} & \multirow[t]{2}{*}{$\begin{array}{l}\text { ZAP-70 } \\
\text { protein }\end{array}$} & \multicolumn{4}{|c|}{$\begin{array}{c}\text { Cytogenetic abnormalities } \\
\text { ( } \% \text { cells })\end{array}$} & \multirow{2}{*}{$\begin{array}{c}\text { Birth } \\
\text { ratec }^{\mathrm{C}} \\
\text { (\%/day) }\end{array}$} \\
\hline & & & & & & & & & & $\Delta 11 q 22$ & Tri12 & $\Delta 13 q 14$ & $\Delta 17 p 23$ & \\
\hline CLL107 & 60 & M & 0 & 8.25 & - & - & 6.9 & 1 & - & ND & $<5$ & 21.1 & $<5$ & 0.23 \\
\hline CLL109 & 76 & $\mathrm{~F}$ & $0 / 1$ & 9.0 & - & - & 7.1 & 21 & - & ND & 59.7 & $<5$ & $<5$ & 0.11 \\
\hline CLL165 & 64 & M & 0 & 5.5 & - & - & 5 & 2 & - & 38.3 & $<5$ & 96 & $<5$ & 0.14 \\
\hline CLL169 & 40 & M & I & 6.0 & + & Post 2 yr & 5.1 & 5 & - & ND & $<5$ & 90.9 & $<5$ & 0.49 \\
\hline CLL189 & 58 & M & IV & 5.0 & + & $\begin{array}{l}\text { Pre } 1 \mathrm{yr} / \\
\text { Post } 0.3 \mathrm{yr}\end{array}$ & $0.3,6.8$ & 4 & - & $<5$ & $<5$ & 70 & $<5$ & 0.81 \\
\hline CLL280 & 63 & M & 0 & 3.25 & - & - & ND & 1 & + & $<5$ & $<5$ & 98.6 & $<5$ & 0.22 \\
\hline CLL282 & 65 & M & II & 2.0 & + & $\begin{array}{l}\text { Pre } 1.5 \mathrm{yr} / \\
\text { Post } 1.5 \mathrm{yr}\end{array}$ & 2.4 & 0 & + & ND & 100 & $<5$ & $<5$ & 0.39 \\
\hline CLL321 & 50 & M & 0 & 5.0 & - & - & 0.3 & 6 & ++ & $<5$ & 58.9 & $<5$ & $<5$ & 0.29 \\
\hline CLL331 & 71 & M & 0 & 0.75 & - & - & 3.7 & 1 & - & $<5$ & $<5$ & $<5$ & $<5$ & 0.24 \\
\hline CLL332 & 46 & M & I & 0.5 & + & Post 1 yr & 1 & 57 & + & ND & $<5$ & 93.5 & $<5$ & 0.24 \\
\hline CLL336 & 74 & $\mathrm{~F}$ & IV & 4.75 & + & Post 2 yr & 2 & 2 & - & ND & $<5$ & 89.5 & $<5$ & 0.41 \\
\hline CLL355 & 71 & M & I & 5.0 & + & - & 0 & 24 & - & 68.4 & $<5$ & 25 & $<5$ & 0.45 \\
\hline CLL360 & 60 & M & $0 / 1$ & 2.0 & - & - & 0.3 & 32 & ++ & ND & 7.5 & 7.5 & $<5$ & 0.48 \\
\hline CLL394 & 55 & M & I/II & 1.0 & - & - & 0 & 72 & ++ & 76.2 & $<5$ & 55.6 & $<5$ & 0.18 \\
\hline CLL400 & 63 & M & 1 & 1.0 & + & $\begin{array}{l}\text { Pre } 1 \mathrm{yr} / \\
\text { Post } 0.5 \mathrm{yr}\end{array}$ & 0 & 5 & ++ & $<5$ & $<5$ & $<5$ & $<5$ & 1.76 \\
\hline CLL403 & 49 & M & II & 2.5 & + & Post $0.25 \mathrm{yr}$ & 0 & 68 & + & 90.5 & $<5$ & 11.3 & $<5$ & 0.39 \\
\hline CLL408 & 63 & M & I A & At diagnosis & - & - & 0.3 & 98 & + & $<5$ & $<5$ & 23.3 & $<5$ & 1.09 \\
\hline CLL418 & 67 & M & 0 & 0.25 & - & - & 4 & 17 & - & ND & 47.4 & 45.6 & $<5$ & - \\
\hline CLL472 & 67 & M & 0 & 0.25 & + & - & 0 & 9 & ++ & $<5$ & 40 & $<5$ & $<5$ & 0.54 \\
\hline
\end{tabular}

\footnotetext{
ATemporal relationship, in years, between treatment initiation and current study. BPercent difference from most similar germ-line gene. DNA sequences at least $2 \%$ different from the most similar germ-line counterpart were considered mutated. CPercent values apply to the current study. ND, not determined; pre, treatment given before beginning of study; post, treatment started after beginning of study; Tri12, trisomy 12; $\Delta 11 q 22$, deletion at 11q22; $\Delta 13 q 14$, deletion at $13 q 14 ; \Delta 17 \mathrm{p} 23$, deletion at $17 \mathrm{p} 23$.
}

This study used deuterium, a nonradioactive isotope detectable by mass spectrometry, that was administered in the form of deuterated "heavy" water $\left({ }^{2} \mathrm{H}_{2} \mathrm{O}\right)$, to label newly synthesized DNA of dividing cells in vivo (26). The kinetic profiles identified for many patients fit first-order assumptions, although in other patients more complexity was observed and the use of a 2-compartment model and important analytical corrections were required. The data demonstrate that B-CLL cells proliferate at definable and, in some cases, substantial rates (about $0.1 \%$ to greater than $1.0 \%$ of the clone per day). In most instances, patients whose clonal birth rates exceeded $0.35 \%$ per day had symptoms or signs of active or progressive B-CLL. Thus, these studies highlight the dynamic nature of B-CLL clones and define sizeable rates of birth and death that were previously unappreciated. The rates of daily clonal turnover may serve as an additional indicator of patients at risk for more aggressive forms of this currently incurable disease.

\section{Results}

Measurement of ${ }^{2} \mathrm{H}_{2} \mathrm{O}$ enrichment in vivo. Nineteen patients were enrolled in the current study (Table 1). Patients daily drank sufficient ${ }^{2} \mathrm{H}_{2} \mathrm{O}$ (approximately $60 \mathrm{ml} / \mathrm{d}$ ) to reach a systemic level of ${ }^{2} \mathrm{H}_{2} \mathrm{O}$ of approximately $1-2 \%$ of total body water. This dose is diluted into the body water pool - whose size, as a fraction of body weight, was estimated from the rate of ${ }^{2} \mathrm{H}$ loss after the ${ }^{2} \mathrm{H}_{2} \mathrm{O}$ intake period (see Methods and Figure 1A). Figure 1B illustrates ${ }^{2} \mathrm{H}_{2} \mathrm{O}$ enrichments achieved in the aqueous fraction of plasma and in DNA of polymorphonuclear leukocytes (PMNLs) from patient CLL165 after an 84-day labeling phase and a 104day washout period. These data are typical for other patients in the study. Note that PMNLs with ${ }^{2} \mathrm{H}$-marked DNA were identified in the blood approximately 7 days after the patient began imbibing ${ }^{2} \mathrm{H}_{2} \mathrm{O}$. Peak enrichment for these cells was reached by 14 days and was maintained until cessation of ${ }^{2} \mathrm{H}_{2} \mathrm{O}$ intake, at which time marked cells began to decline. The results obtained for PMNLs provided a kinetic comparison of a normal and rapidly-turning-over cell population with the leukemic B lymphocytes of each patient.

Maximum levels of ${ }^{2} \mathrm{H}_{2} \mathrm{O}$ were detected in the plasma of this and other patients at the time of the first blood drawing, day 7 (Figure 1B). This equilibrium level was maintained throughout the 84-day labeling period and then decreased after ${ }^{2} \mathrm{H}_{2} \mathrm{O}$ intake ceased. Close inspection of the washout phase revealed that it could take more than 2 weeks for the body ${ }^{2} \mathrm{H}_{2} \mathrm{O}$ levels to drop to $50 \%$ and over 5 weeks for them to drop to $10 \%$ of equilibrium values (Figure $1 \mathrm{~B}$ ). To account for this persistence and to assure that residual ${ }^{2} \mathrm{H}$ present during the washout phase did not skew interpretation of subsequent data points, an exponential model for body ${ }^{2} \mathrm{H}_{2} \mathrm{O}$ levels was developed (see Methods). The body ${ }^{2} \mathrm{H}_{2} \mathrm{O}$ model predicted that the uncorrected skewing could be significant for certain subjects; in particular, those individuals whose daily water exchange rates $\left(f_{\mathrm{w}}\right)$ were low (Figure 1C). This was the case for several patients in this study (see Supplemental Table 1; supplemental material available online with this article; doi:10.1172/JCI200523409DS1) and is not surprising since B-CLL patients are often elderly and less active. The model accurately predicted the measured levels of body 

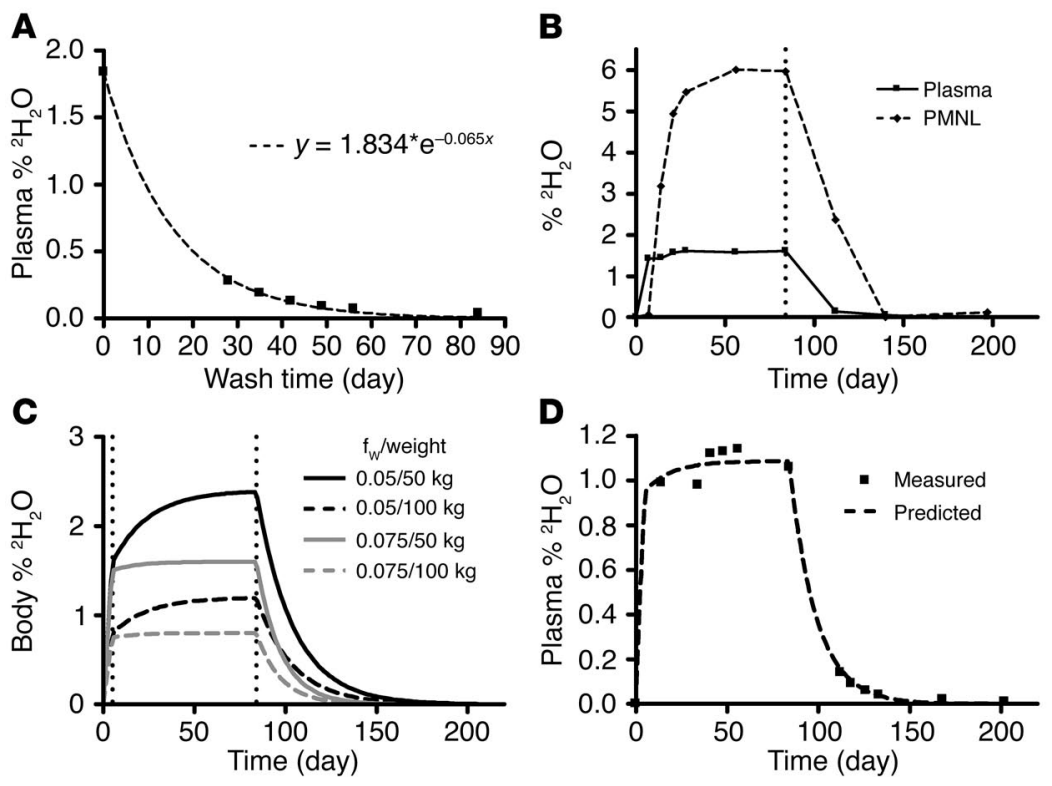

Figure 1

Modeling of the ${ }^{2} \mathrm{H}_{2} \mathrm{O}$ levels in patients drinking ${ }^{2} \mathrm{H}_{2} \mathrm{O}$. (A) ${ }^{2} \mathrm{H}_{2} \mathrm{O}$ enrichment data from the plasma of a CLL patient, showing that the dilution of ${ }^{2} \mathrm{H}_{2} \mathrm{O}$ during the washout phase fits an exponential decay model. For the patient shown (CLL109), the equilibrium ${ }^{2} \mathrm{H}_{2} \mathrm{O}$ enrichment was approximately $1.8 \%$ during the ${ }^{2} \mathrm{H}_{2} \mathrm{O}$ intake period. Therefore, the daily water exchange rate, $W_{\text {day }}$, for this individual was approximately $2.3 \mathrm{l} / \mathrm{d}\left({ }^{2} \mathrm{H}_{2} \mathrm{O}\right.$ intake of $60 \mathrm{ml} / \mathrm{d} \times 70 \%{ }^{2} \mathrm{H}_{2} \mathrm{O}=42 \mathrm{ml} / \mathrm{d}$, divided by 0.018 ). The rate of the exponential decay is the fractional daily water exchange rate, $f_{\mathrm{w}}$, and, for the patient shown, was 0.065 per day. The total body water was therefore 35.4 liters (2.3/0.065). This individual weighed $55 \mathrm{~kg}$, so the fraction of body weight due to water was 0.64 . This fraction was used for all patients in the study to estimate total body water when a patient's weight was available. When a patient's weight was not recorded, an $f_{\mathrm{w}}$ value of $6 \%$ per day was used. (B) Representative raw data for ${ }^{2} \mathrm{H}_{2} \mathrm{O}$ enrichment in plasma and $\mathrm{CD} 5{ }^{+} \mathrm{CD} 19^{+}$cells from a B-CLL patient (CLL165). PMNL DNA saturates at a stable enrichment related to the ${ }^{2} \mathrm{H}_{2} \mathrm{O}$ plasma enrichment. The vertical line at day 84 indicates the time at which the patient ceased intake of ${ }^{2} \mathrm{H}_{2} \mathrm{O}$. (C) Body ${ }^{2} \mathrm{H}_{2} \mathrm{O}$ enrichment models for patients of various weights and fractional daily water exchange rates. Patients with high $f_{w}$ equilibrate faster, while patients with low $f_{w}$ can deviate substantially from a square pulse. (D) Body ${ }^{2} \mathrm{H}_{2} \mathrm{O}$ enrichment model derived from a patient's weight and equilibrium ${ }^{2} \mathrm{H}_{2} \mathrm{O}$ enrichment accurately predicts the measured body ${ }^{2} \mathrm{H}_{2} \mathrm{O}$ enrichment values.

${ }^{2} \mathrm{H}_{2} \mathrm{O}$ in the subjects studied (Figure 1D). Therefore, we incorporated this ${ }^{2} \mathrm{H}_{2} \mathrm{O}$ function into the models used (Figure 2) for estimating cell kinetics during the washout phase.

Kinetic patterns of leukemic B lymphocytes in individual B-CLL patients. The fraction of labeled B-CLL cells for the 19 patients at each time point of their involvement in the study is shown in

\section{Figure 2}

Models for analysis of cellular ${ }^{2} \mathrm{H}$ enrichment. (A) Single-compartment model in which 1 well-mixed pool is assumed. Unequal birth and death rates cause a change in pool size, but the labeled cell fraction is a function only of the birth rate (see Methods). (B) Two-compartment model in which cells proliferate in a distinct compartment $\left(v_{1}\right)$ and efflux into the sampled compartment (blood, $v_{2}$ ). The birth, efflux, and death rates in all compartments were assumed to be equal. This model does not explicitly allow for the return of cells from the blood to the first compartment, but a high rate of exchange between the compartments relative to the birth rate would functionally equilibrate the model into a single compartment. Total clonal birth rate, $B$, is $b / 1+v_{\mathrm{r}}$, where $v_{\mathrm{r}}$ is the size of the second compartment relative to the first $\left(v_{2} / v_{1}\right)$.
Figure 3. White blood cell (WBC) counts are also plotted for each patient during the study period. It is noteworthy that the patterns of appearance and disappearance of ${ }^{2} \mathrm{H}$-marked cells varied among patients, although shared qualitative features were apparent.

First, patients could be divided as having high birth rates, with fractions of labeled cells exceeding 0.35 at the peak (patients CLL169, CLL189, Cll282, Cll336, Cll355, Cll360, Cll400, CLL403, and CLL472), or low birth rates, with fractions less than this value (Figure 3). Second, in most instances, labeled B-CLL cells appeared in the blood within a few weeks, which indicates that the cells are rapidly released from the site of division following new DNA synthesis and cell division. There were, however, exceptions. In 6 patients (CLL107, CLL165, CLL280, CLL355, CLL394, and CLL403), there was a significant lag between initiation of ${ }^{2} \mathrm{H}_{2} \mathrm{O}$ intake and appearance of labeled cells in the peripheral blood. In these subjects, enrichment of ${ }^{2} \mathrm{H}$ in cellular DNA continued to rise after the 84 -day ${ }^{2} \mathrm{H}_{2} \mathrm{O}$ intake period was completed. This was most pronounced in patient CLL355 (Figure 3), in whom no marked cells appeared in the blood until 56 days into the labeling period and peak enrichment did not occur until approximately 155 days into the study period (71 days after ${ }^{2} \mathrm{H}_{2} \mathrm{O}$ intake ceased). No changes in lymph node or spleen size were found on physical examination in this patient. Third, the decline phase was quite heterogeneous among patients, with examples of apparent fast (e.g., patients CLL189 and CLL400), slow (e.g., patients CLL107, CLL331, and CLL418), and negligible (e.g., patients CLL165, CLL332, and CLL394) elimination functions (Figure 3).

$B$ irth rates of $B$-CLL cells in vivo. The qualitative descriptions above are limited in their utility, and more quantitative data were desired. Therefore,

${ }^{2} \mathrm{H}$ enrichment data obtained for each patient's $\mathrm{CD}^{+} \mathrm{CD} 19^{+} \mathrm{B}$ cells were analyzed in 3 ways (Table 2 and Supplemental Table 1). First, a minimum rate of cell proliferation in the labeled cell fractions was calculated by division of the peak level

A

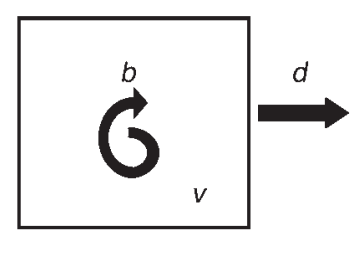

B

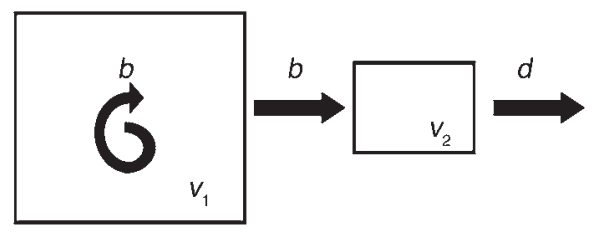



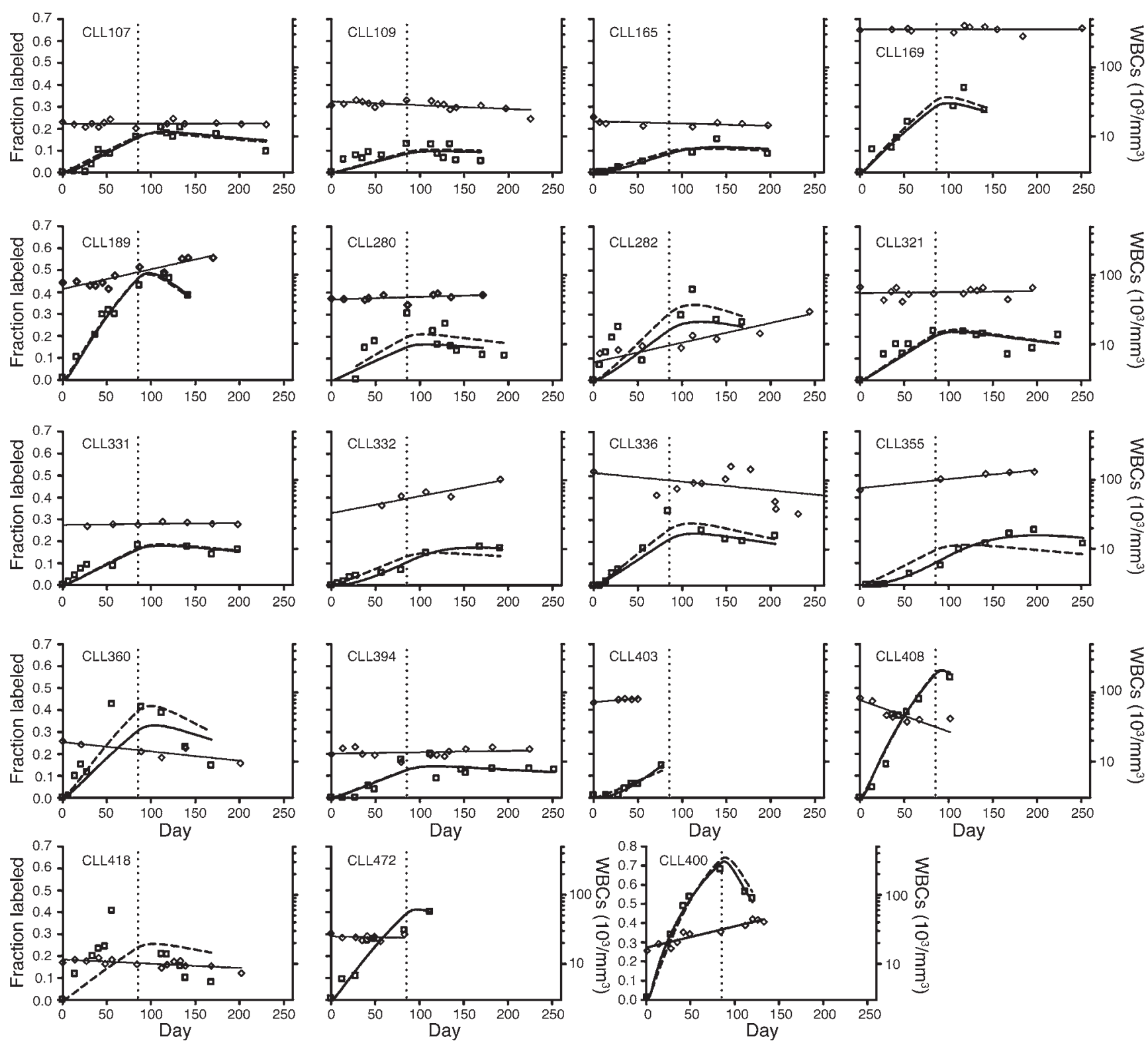

Figure 3

Labeling data, model fits, and WBC counts for the $19 \mathrm{~B}-\mathrm{CLL}$ patients studied. Percent ${ }^{2} \mathrm{H}$ enrichment in the DNA of leukemic cells was measured and converted into a fraction of labeled cells as described in Methods (squares, left axis). The single-compartment (dashed line) and 2-compartment (solid line) models were fit to the data using nonlinear curve fitting software. WBC counts taken during the study are shown (diamonds) plotted on a log scale at right. An exponential growth function was fit to the WBC data to provide a growth rate for the leukemic cells in the blood (Table 2).

of the fraction of labeled cells $(f)$ by the duration of water intake. The peak levels of $f$ ranged from 0.13 (patient CLL109) to 0.68 (patient CLL400). These calculations indicated leukemic cell proliferation rates between $0.10 \%$ and $0.81 \%$ per day $\left(f_{\text {peak }} \% /\right.$ day, Table 2). Preliminary analyses of peripheral B cells from 3 normal individuals matched for age (50-72 years), who followed the same ${ }^{2} \mathrm{H}_{2} \mathrm{O}$ drinking protocol, indicated $f_{\text {peak }} \% /$ day values between $0.1 \%$ and $0.3 \%$ (data not shown).

Next, we used single-compartment (Figure 2A) and 2-compartment (Figure 2B) models to fit to the data. Both approaches take advantage of all the data obtained at each of the multiple time points sampled. The latter approach may have additional merit since it anticipates that B-CLL cells proliferate in a distinct compartment, i.e., the solid lymphoid tissues, separated from the sampled blood compartment.

Both models were fit to the data of all patients (Figure 3), and the resulting parameter values were determined (Table 2 and Supplemental Table 1). Using these 2 approaches, birth rates of leukemic cells from the 19 patients studied were similar and ranged from $0.12 \%$ to $1.08 \%$ per day for the single-compartment model and from $0.11 \%$ to $1.75 \%$ per day for the 2 -compartment model. Thus, for most patients, the 2 models did not differ appreciably in quality of fit to the data or in the resulting birth rate estimates, and they were therefore interchangeable (Table 2 
Table 2

Kinetic and model parameters

\begin{tabular}{|c|c|c|c|c|}
\hline Patient & $f_{\text {peak }} \%$ & $\boldsymbol{t}_{\text {peak }} \% /$ day & $\begin{array}{c}\text { Single- } \\
\text { compartment } \\
\text { model, } \boldsymbol{b} \% / \text { day }^{\mathrm{A}}\end{array}$ & $\begin{array}{c}\text { Two- } \\
\text { compartment } \\
\text { model, } B \% / \text { day }\end{array}$ \\
\hline CLL107 & 20.7 & 0.25 & 0.22 & 0.23 \\
\hline CLL109 & 13.1 & 0.16 & 0.12 & 0.11 \\
\hline CLL165 & 15.2 & 0.18 & 0.13 & 0.14 \\
\hline CLL169 & 38.6 & 0.46 & 0.49 & 0.49 \\
\hline CLL189 & 43.1 & 0.51 & 0.80 & 0.81 \\
\hline CLL280 & 30.4 & 0.36 & 0.22 & 0.22 \\
\hline CLL282 & 41.3 & 0.49 & 0.55 & 0.39 \\
\hline CLL321 & 22.5 & 0.27 & 0.30 & 0.29 \\
\hline CLL331 & 18.2 & 0.22 & 0.24 & 0.24 \\
\hline CLL332 & 17.6 & 0.21 & 0.18 & 0.24 \\
\hline CLL336 & 34.0 & 0.40 & 0.40 & 0.41 \\
\hline CLL355 & 25.1 & 0.30 & 0.24 & 0.45 \\
\hline CLL360 & 41.4 & 0.49 & 0.66 & 0.48 \\
\hline CLL394 & 19.9 & 0.24 & 0.17 & 0.18 \\
\hline CLL400 & 68.1 & 0.81 & 1.73 & 1.76 \\
\hline CLL403 & 14.6 & 0.17 & 0.17 & 0.39 \\
\hline CLL408 & 54.8 & 0.65 & 1.08 & 1.09 \\
\hline CLL418 & 40.7 & 0.48 & 0.28 & - \\
\hline CLL472 & 31.8 & 0.38 & 0.53 & 0.54 \\
\hline
\end{tabular}

Altalicized values indicate poor model fits.

and Supplemental Table 1). However, the 2-compartment model fit the data of patients CLL332, CLL355, and CLL403 much more accurately (see $r^{2}$ values in Supplemental Table 1), and the birth rate estimates were larger based on the 2-compartment model fits for these 3 patients ( 0.24 vs. $0.18 \%$ /day, 0.45 vs. $0.24 \%$ /day, and 0.39 vs. $0.17 \% /$ day, respectively). The data from 4 patients (CLL109, CLL280, CLL282, and CLL418) did not fit well into either model.

Long-term follow-up measurements. Four patients (CLL165, CLL331, CLL336, and CLL355) provided blood samples more than 1 year after the completion of the original study $(624,519,666$, and 444 days after discontinuing ${ }^{2} \mathrm{H}_{2} \mathrm{O}$ intake, respectively). When circulating B-CLL cells were isolated, significant levels of ${ }^{2} \mathrm{H}$ were still detectable in the DNA of the leukemic cells (Figure 4). The 2-compartment models that had been fit to their original data were projected out to the time of the acquisition of these longterm samples, and in each case the labeled cell fractions were quite close to the values expected by the model projections.

Growth and death rates of B-CLL cells in vivo. Clonal growth rates were calculated for each patient by fitting of an exponential curve (Figure 3 and Table 3 ) to the WBC counts from the beginning to the end of the study period. Five patients (CLL189, CLL332, CLL355, CLL400, and CLL403) had increasing numbers of WBCs and thus positive growth rates, 6 patients (CLL107,
CLL169, CLL321, CLL331, CLL394, and CLL472) had stable WBC levels and essentially zero growth rates, and 4 patients (CLL165, CLL336, CLL360, and CLL408) with falling WBC counts had negative growth rates. By assuming that the rate of change of the peripheral blood WBC count reflects total clonal growth (in the absence of identifiable changes in the size of measurable peripheral tissues), we calculated a death rate for each patient's clone as the birth rate minus the growth rate (Table 3 ).

Interestingly, there was not an obvious correlation between birth rates and growth rates, presumably because of differences in death rates (Figure 5). For example, patient CLL408 had the second highest birth rate of any individual in the study $(1.09 \%$ per day), although the WBC number actually fell during the course of the study. Patients CLL355 and CLL400 had positive growth rates and increasing WBC counts. For patient CLL355, the birth rate was approximately equal to the growth rate, suggesting little death, whereas patient CLL400 had a birth rate that exceeded the growth rate, suggesting significant cell death.

Relationship between in vivo B-CLL cell kinetics and disease activity and progression. Of the 19 patients enrolled in this study, 10 had B-CLL cell birth rates greater than $0.35 \%$ of the clone per day (Table 2 ). Of these patients, 8 were designated as having active or progressive disease based on criteria defined by the National Cancer Institute (Table 1) (27). In contrast, for those 9 patients whose leukemic cells had birth rates less than $0.35 \%$, only 1 was classified as having active or progressive disease. Thus there appears to be a direct and statistically significant ( $P=0.0055$ by Fisher's exact test) relationship between leukemic cell birth rates and a patient's clinical course. Nevertheless, a statistically significant correlation between the in vivo kinetic data and differences in $\mathrm{Ig} \mathrm{V}_{\mathrm{H}}$ gene mutation status, ZAP-70 or CD38 expression, and cytogenetic abnormalities was not defined (Tables 1 and 2).
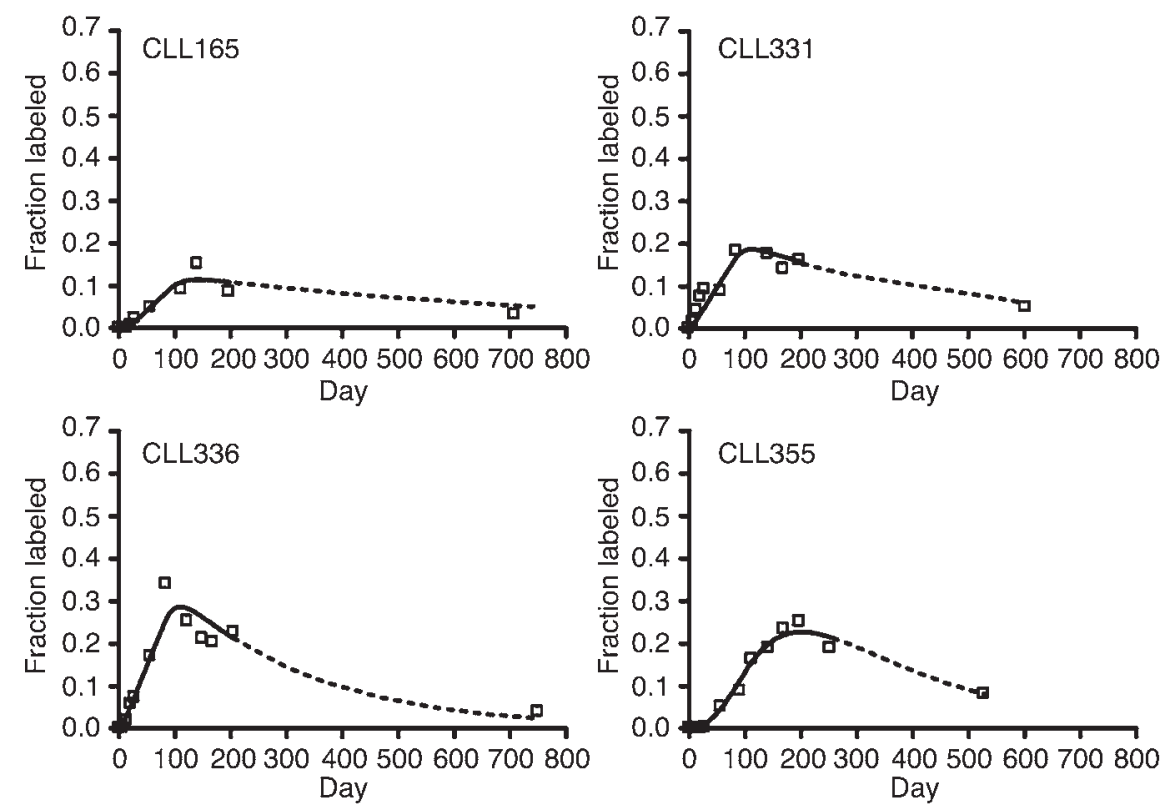

\section{Figure 4}

Long-term follow-up samples are consistent with model assumptions. Samples from 4 patients were obtained more than a year after the end of the study. The labeled cell fractions were very close to the values predicted by the projected (dashed line) 2-compartment models that fit the earlier data (solid line). 
Table 3

Growth and death rates

\begin{tabular}{cccc} 
Patient & $\Delta$ WBC \%/day & $\begin{array}{c}\text { Single-compartment } \\
\text { model, death, } \\
\% / \text { day }\end{array}$ & $\begin{array}{c}\text { Two-compartment } \\
\text { model, death, } \\
\text { \%/day }\end{array}$ \\
CLL107 & 0.006 & 0.21 & 0.22 \\
CLL109 & -0.125 & 0.25 & 0.24 \\
CLL165 & -0.073 & 0.20 & 0.21 \\
CLL169 & -0.001 & 0.49 & 0.49 \\
CLL189 & 0.656 & 0.14 & 0.15 \\
CLL280 & 0.087 & 0.13 & 0.13 \\
CLL282 & 0.654 & -0.10 & -0.26 \\
CLL321 & 0.035 & 0.27 & 0.26 \\
CLL331 & 0.033 & 0.21 & 0.21 \\
CLL332 & 0.567 & -0.39 & -0.33 \\
CLL336 & -0.289 & 0.69 & 0.70 \\
CLL355 & 0.302 & -0.06 & 0.15 \\
CLL360 & -0.311 & 0.97 & 0.79 \\
CLL394 & 0.045 & 0.13 & 0.14 \\
CLL400 & 0.712 & 1.02 & 1.05 \\
CLL403 & 0.241 & -0.07 & 0.15 \\
CLL408 & -1.052 & 2.13 & 2.14 \\
CLL418 & -0.141 & 0.42 & - \\
CLL472 & -0.053 & 0.58 & 0.59 \\
\hline
\end{tabular}

Altalicized values indicate poor model fits.

\section{Discussion}

In this study, we identified newly formed leukemic B cells by using an in vivo stable isotope labeling technique (26). This technique utilizes ${ }^{2} \mathrm{H}_{2} \mathrm{O}$, which is an easily administered, well-tolerated, and harmless molecule that can be taken for weeks or months. B-CLL cells were analyzed for ${ }^{2} \mathrm{H}$ incorporation into deoxyribose in DNA in order to measure cell division. From these data, we made several informative observations regarding the birth, growth, and death rates of B-CLL cells in vivo.

Birth rates of B-CLL cells. The leukemic cells of patients with B-CLL do not have inconsequential levels of cell division in vivo, as previously hypothesized (5). Rather, ample cellular proliferation clearly occurs within the leukemic clones of all B-CLL patients, and in some patients these levels are quite substantial (Table 2). For example, for subject CLL400, 68.1\% of the B-CLL cells were newly synthesized over the 84 -day labeling period $\left(f_{\text {peak }}\right.$, Table 2$)$. In contrast, the leukemic cells of some subjects (e.g., patient CLL109) exhibited much slower, but still significant, levels of cell proliferation, with approximately $13 \%$ being born during the same labeling interval $\left(f_{\text {peak }}\right.$, Table 2$)$. Presumably, much of the cellular generation emanated from proliferation centers that are frequently found in lymph nodes and bone marrow of B-CLL patients $(3,28)$. It has been suggested that the number and size of these structures correlate with lymphocyte doubling times (29).

Studies performed over 3 decades ago estimated similar daily proliferation rates $(30,31)$, although these studies were hindered by difficulties inherent in using radioactive tracers in humans, the need to perform ECIB, and the inability to precisely define leukemic lymphocytes within blood mononuclear cell fractions. Nevertheless, the fact that these approaches yielded data in line with those reported here supports our findings, since each methodology is prone to distinct potential artifacts. The method used here, labeling newly synthesized DNA, might be influenced if there were very substantial levels of DNA synthesis not associated with replication (i.e., DNA repair, sometimes termed "unscheduled DNA synthesis"). However, if this were the case, then the data obtained using ECIB or tracking of chromium-labeled cells, which do not require the incorporation of a label into newly synthesized DNA, would not correlate with our data or the old data generated using the ${ }^{3} \mathrm{H}$ infusion technique that also involves an incorporation process. Furthermore, although normal B lymphocytes have a relatively high level of ongoing DNA repair (32), there is no clear-cut evidence for increased basal DNA repair activity in B-CLL cells. Finally, if increased unscheduled DNA synthesis were responsible for incorporation of significant amounts of label, one would expect that every subject would show a steady increase in labeled DNA from the beginning of the intake period, which was clearly not the case for several of our subjects.

Variability in patterns of leukemic cell turnover among B-CLL patients. Another intriguing observation is the diversity in the patterns of appearance and disappearance of ${ }^{2} \mathrm{H}$-marked B-CLL cells in the blood of different patients. This variability includes a marked lag in appearance of ${ }^{2} \mathrm{H}$-marked B-CLL cells in the blood for some subjects (Figure 3). As would be expected in these patients, the fraction of labeled cells continued to increase for many weeks after the end of the ${ }^{2} \mathrm{H}$ intake phase. Since those cells appearing after intake must have been produced while ${ }^{2} \mathrm{H}$ was present, they had to have remained in a separate compartment for some time prior to being released into the blood.

The analysis of B-CLL cell kinetics must consider potential trafficking of cells between various body compartments. Several studies have indicated the presence of an extravascular compartment that rapidly equilibrates with blood, so that these two can be considered as a single anatomic unit (33). The concept of the existence of a second compartment, engaged in a lesser level of exchange with the peripheral compartment, came from results of studies of ECIB (30) and chromium infusion that suggested that this compartment probably included the spleen (34). Finally, a less exchangeable compartment, probably organized lymphoid tissue and bone marrow, was postulated $(30,34)$ to contain the vast majority of leukemic cells not accounted for by the other two (35). These compartments were simplistic in biologic terms, and,

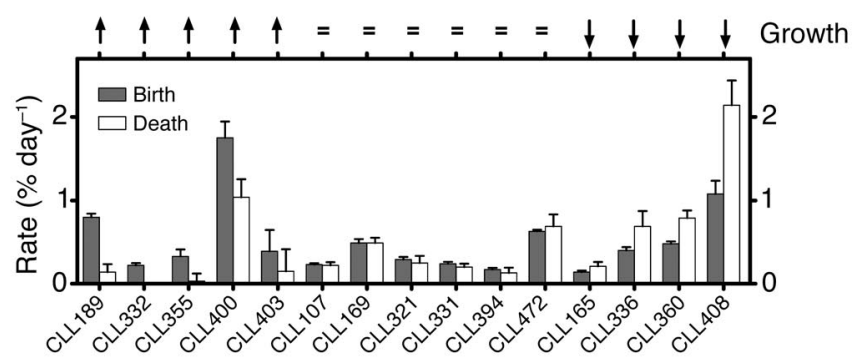

Figure 5

Birth and death rates of B-CLL cells. The total clonal birth rates, $B$, are depicted in gray. The values were calculated from the 2-compartment model fitting parameters $b$ and $v_{r}$ (see Methods) for those patients in which the $r^{2}$ value of the model fit was greater than 0.6. The error bars represent SEs of the estimates. The growth rate trends are indicated across the top, as derived from Figure 3 and Table 2. The death rates were calculated by subtraction of the growth rate from the birth rate. The SE of the death rates was calculated from the square root of the sum of squares of the SE of the total clonal birth rates and the SE of the growth rate. 
in addition, their conceptualization led to models of B-CLL kinetics that were mathematically complex and difficult to apply to data obtained only from peripheral cells.

Because of such issues (lag in the appearance of labeled cells in the blood and the possibility of trafficking/recirculation complexities), we chose to analyze our data using both a 2-compartment model, consisting of the sampled blood compartment and a proliferative compartment, and a single-compartment model that combines these 2 sites. The single-compartment model fit many patients as well as the 2-compartment model did, even though the latter was anticipated to more accurately represent the biologic reality. In these cases, the birth rate estimates were similar to the minimal birth rate estimate of the peak $f$ divided by the duration of ${ }^{2} \mathrm{H}_{2} \mathrm{O}$ consumption. Explanations may include a high rate of exchange, relative to cell birth, between compartments or a very large proliferative compartment relative to the sampled compartment. The relative sizes of the compartments $\left(v_{\mathrm{r}}\right)$ predicted by the 2-compartment model (Supplemental Table 1) were consistent with the accepted notion that the bulk of the leukemic clone resides in solid lymphoid organs. The data from several patients (CLL332, CLL355, and CLL403) were significantly better fit by the 2-compartment model; this justified its use and pointed to variations in the kinetic structure between and possibly within B-CLL clones. Of note, CLL332 was modeled to have a birth rate that was less than the WBC growth rate. This inconsistency suggests an additional level of biologic complexity not accounted for in our models, but potentially of physiologic interest.

There are several assumptions implicit in the models we used. The compartments were assumed to be homogeneous and well mixed with no significant subcompartmental sequestering or structure. The 2-compartment model assumes that changes in WBC counts reflect those of the total clone, which may not necessarily be the case in every patient. Furthermore, the rates of birth, efflux, and death were assumed to remain constant without bursts of growth or release from the first compartment or sudden die-offs or clearance from the blood. The long-term follow-up data from the 4 patients illustrated in Figure 4 suggest that the measured rates can remain stable, and therefore, at least for these patients, the assumptions were not grossly inaccurate. Considerable deviations from these predicted values may be indicative of changes in the kinetic properties of the clone or its members.

Death rates of $B$-CLL cells. Finally, the proliferation rates that we have documented, particularly in patients with stable WBC counts, suggest that the B-CLL clone is continually dying and replenishing itself. Indeed, we calculated death rates approaching or exceeding $1 \%$ of the entire clone per day in some patients (e.g., CLL336, CLL360, CLL400, CLL408, and CLL472; Table 2 and Figure 5). Thus, in most patients, a significant portion of the clone is not inherently defective in apoptotic potential, since, if such a defect were present in all members of a B-CLL clone, stable WBC counts would not be observed in the presence of cell proliferation. It is interesting that the fastest clonal birth was not always associated with increases in WBC counts, at least during the study period. For example, patient CLL169 exhibited a massive circulating clonal load (approximately 350,000 cells per cubic millimeter) that remained stable over the 6-month experiment, and patient CLL336 exhibited a clonal fall of an appreciable degree (134,000 per milliliter to 49,300 per milliliter). Both patients exhibited high fractions of newly divided B-CLL cells (Figure 3 and Table 2), and in each case, there were no detect- able changes in clinical parameters. These findings emphasize that stability in blood WBC levels does not reflect underlying cellular dynamics; rather, the net effect of clonal turnover at the WBC level reflects a balance between cell birth and death rates. Although in most instances of malignancy a tendency for higher birth or lower death rates is presumably present, there may be times in the evolution of a B-CLL clone when these two are equal or even tipped toward slower growth or faster death, in at least a portion of the clone (Figure 5). Such shifts in net balance may explain the clinical observation that WBC levels can increase over time in a stepwise, as opposed to linear, fashion.

Clinical implications. The measurements presented here provide a foundation for kinetic modeling of this disease, which may predict clonal behavior, explain clinical observations, and suggest therapeutic approaches. The measurement of clonal birth and death rates may provide another manner by which clinical progression could be defined and even predicted. The correlation between birth rates and disease activity/progression suggests that this may very well be true. Eighty percent of those patients with clonal birth rates in excess of $0.35 \%$ per day exhibited evidence for active or progressive disease in the 6-month period preceding the study or in the subsequent follow-up period after leaving the study. Furthermore, 5 of the 6 patients that required therapy before, during, or after the study period had birth rates greater than $0.35 \%$ per day. Despite this relationship, a significant correlation between the in vivo kinetic data and differences in $\mathrm{Ig} \mathrm{V}_{\mathrm{H}}$ gene mutation status, ZAP-70 or CD38 expression, cytogenetic abnormalities, or serum lactate dehydrogenase, or differences in findings on physical examination (i.e., lymphadenopathy or splenomegaly), was not identified. However, this may represent the fact that the latter markers portend the eventual clinical outcome for an individual patient, whereas the former measurements are "real-time" estimates of the kinetic activities of the leukemic clones. A larger study will be necessary to determine whether these correlations persist.

In addition, the ability to analyze a patient's ${ }^{2} \mathrm{H}$-labeled cells a year or more after the end of the ${ }^{2} \mathrm{H}_{2} \mathrm{O}$ labeling period (Figure 4) may allow detection of significant kinetic changes in the clone over that period. If the level of enrichment defined at the time of the extended blood sampling were in line with preceding kinetic estimates, then this would suggest a stable clonal structure. However, if the level were significantly lower or higher than predicted, this could reflect either enhanced dilution of the labeled leukemic cells by a faster-growing or slower-dying clonal variant (if lower than predicted) or a deficiency of dilution because of the emergence of variants with decreased birth or enhanced death rates (if higher than predicted). The threshold level of label required for accurate quantification is primarily a function of the mass spectrometry, and more sensitive instruments currently available could significantly extend the post $-{ }^{2} \mathrm{H}_{2} \mathrm{O}$-drinking period in which useful data could be obtained.

The birth rates described here indicate that there must be a discernible level of cell division occurring in all patients at all times. This reservoir of dividing leukemic cells, which replenishes, maintains, and augments clone size, may be prime targets of therapeutic agents directed toward dividing cells. Although our studies suggest that only a small fraction of the cells are proliferating at any given time, these dividing cells probably represent the main feeder population for clonal maintenance, growth, and diversification. This is consistent with the findings that active progressive disease correlates with higher birth rates (Tables 1 and 2). 
Finally, a substantial birth rate also enables consideration of models of disease progression that invoke clonal evolution as a significant event. The rate at which a biologic system evolves is determined by the frequency at which variants are produced and their relative selective advantages. For newly generated subclones to become a major proportion of the total population, they must either arise very early in the life of the clone or have a selective advantage over the bulk of the clone. Selective advantage manifests as an increased growth rate, which could be due to faster cell division, decreased cell death or clearance, or both. If the B-CLL clone were simply dividing slowly with little loss of cells, it would be difficult for novel variants to catch up with the already massive leukemic burden. However, in the face of substantial proliferation and death of the leukemic cells, novel variants could more quickly expand to a substantial level. Possibly, the growth rate of B-CLL cells may not be as important for prognosis as the birth rate, since proliferation, even if it is counterbalanced by apoptosis, may not result in excessive clonal growth but could facilitate accumulation of new dangerous mutations. The level of B-CLL cell turnover that we have documented, therefore, has important implications for the natural history of an individual patient's disease progression.

In summary, B-CLL cells proliferate at appreciable rates. This finding is in conflict with the dogma that B-CLL is a disease characterized almost exclusively by cell accumulation due to a defect in apoptosis. Indeed, a wide range of cell proliferation and death rates exists among different patients, suggesting that, as in most cancers, the dynamic interplay between these processes may differ among and within patients. The observed interindividual diversity in cellular kinetics suggests that further investigations powered to look for correlations between synthesis rates and clonal biology, subsequent disease progression, and response to therapy are warranted.

\section{Methods}

B-CLL patients. Nineteen patients with B-CLL (17 in Rai stages of II or less and 2 in Rai stage IV) diagnosed by established criteria were enrolled in the study after providing informed consent (Table 1). Of the 19 subjects enrolled, 10 had stable disease and 9 active or progressive disease, using established criteria (27). Sixteen patients had never received treatment. Three patients (CLL189, CLL282, and CLL400) had received treatment more than 6 months before entry into the study. Patient CLL403 had to discontinue the study after 12 weeks because of a need to start chemotherapy. Patients CLL189, CLL282, CLL332, CLL336, and CLL400 were treated after the end of the experimental period. Patient CLL472 did not return after 12 weeks into the study for personal reasons. None of the patients in the study exhibited hemodynamic impairment from either cardiac or renal disease. This study was approved by the North Shore-LIJ Health System's Institutional Review Board and was conducted according to the principles of the World Medical Association Declaration of Helsinki.

Experimental protocol. Patients were given premeasured, individual aliquots of ${ }^{2} \mathrm{H}_{2} \mathrm{O}$ (70\% enriched; Cambridge Isotope Laboratories Inc.). Patients drank $90 \mathrm{ml}$ of ${ }^{2} \mathrm{H}_{2} \mathrm{O}$ twice daily for 5 days (loading) followed by $60 \mathrm{ml}$ once daily for 79 days (maintenance). The goal was to enrich body water to $1-1.5 \%$ deuterium. Patients returned at scheduled intervals during and following the labeling period to donate blood for cell and serum analyses. In addition, all patients kept a daily $\log$ of ${ }^{2} \mathrm{H}_{2} \mathrm{O}$ intake. These logs were evaluated and discussed with each patient on every visit to the North Shore-LIJ Research Institute's General Clinical Research Center to assure compliance with the protocol. No major unexpected side effects were encountered, although 3 patients complained of a transient sense of lightheadedness during the loading phase.
Cell isolations. PBMCs were isolated from the blood of B-CLL patients by density gradient centrifugation through a Ficoll-Hypaque cushion (Amersham Biosciences Corp.). $\mathrm{CD}^{+} \mathrm{T}$ cells were removed from these fractions by positive selection using anti-CD3 microbeads (Miltenyi Biotec Inc.) following the manufacturer's instructions. $\mathrm{CD}^{+} \mathrm{B}$ cells were separated from the CD3- fraction by incubation with anti-CD5 mAb conjugated with PE (BD Biosciences - Immunocytometry Systems) for 20 minutes at $4{ }^{\circ} \mathrm{C}$, washing 4 times in buffer (PBS, $5 \%$ BSA, 2 mM EDTA), and subsequent incubation with anti-PE mAb linked to beads (Miltenyi Biotec Inc.). The $\mathrm{CD}^{+}$fraction, which contained greater than 95\% CD $19^{+}$ cells as assessed by flow cytometry, was centrifuged into a pellet and stored frozen at $-20^{\circ} \mathrm{C}$ until further use.

PMNLs were separated from the red blood cell fraction after density gradient centrifugation of PBMCs (see above). PBS and 3\% dextran were added at equal volumes to the red blood cells, and the cells were incubated for 25 minutes at room temperature. The suspension containing PMNLs was collected and centrifuged; remaining red blood cells were removed using lysis buffer. PMNLs were stored frozen as dry pellets at $-20^{\circ} \mathrm{C}$ until needed.

Analyses of Ig $V_{H}$ mutation status, expression of ZAP-70 and CD38, and cytogenetic abnormalities. Ig variable sequences were obtained and analyzed as previously described (36). CD38 surface expression was determined by flow cytometry (9). ZAP-70 protein expression was determined by Western blotting (37) using the anti-human ZAP-70 mAb clone 2F3.2 (Upstate Biotechnology Inc.) or clone 29 (BD). The percentage of $\mathrm{T}$ cells or NK cells present in each B-CLL sample was determined by immunofluorescence using anti-CD3 or anti-CD56 $\mathrm{mAb}(\mathrm{BD})$. In those samples with greater than $10 \%$ contaminating non-B cells, B lymphocytes were purified by positive selection using anti-CD19 microbeads (Miltenyi Biotec Inc.). B-CLL samples were divided into 3 groups of ZAP-70 expression: ++, 40-100\% positive cells; +, 20-40\%; and -, less than $20 \%$. This percentage was determined by admixture of different concentrations of purified $\mathrm{T}$ cells with mononuclear cells depleted of $\mathrm{T}$ and NK cells from the same healthy donor. Anti-CD3 and anti-CD56 mAb followed by magnetic beads (goat anti-mouse IgG Dynabeads; Dynal Biotech ASA) was used to deplete T and NK cells. Cytogenetic analyses were performed by FISH using the following probes: chromosome 11, LSI ATM at 11q22.3; chromosome 12, CEP-12 centromere; chromosome 13, LSI D13S319 at 13q14.3 and LSI 13q34 at 13q34; and chromosome 17, LSI $\mathrm{p} 53$ at $17 \mathrm{p} 13.1$ (all probes from Vysis Inc.).

Measurement of body ${ }^{2} \mathrm{H}_{2} \mathrm{O}$ enrichment. The enrichment of ${ }^{2} \mathrm{H}_{2} \mathrm{O}$ in plasma was measured by gas chromatography/mass spectrometry (GC/MS) as described previously (26). ${ }^{2} \mathrm{H}_{2} \mathrm{O}$ enrichment was calculated by comparison with standard curves generated by mixture of $100 \%{ }^{2} \mathrm{H}_{2} \mathrm{O}$ with naturalabundance $\mathrm{H}_{2} \mathrm{O}$ in known proportions.

Measurement of stable isotope enrichment in deoxyribose from DNA. Genomic DNA was isolated from B-CLL cells and PMNLs (38) and hydrolyzed to free nucleosides. DNA enrichments were measured by GC/MS. For the majority of samples, free nucleosides were derivatized to a pentane-tetraacetate derivative for GC/MS analysis as described by Neese et al. (26), using selected ion monitoring of mass to charge $(\mathrm{m} / \mathrm{z})$. The isotopic enrichment of $\left[{ }^{2} \mathrm{H}\right]$ deoxyadenosine was determined by comparison with labeled standards.

In some instances, for improved sensitivity with small amounts of DNA, free nucleosides were derivatized with perfluorobenzylhydroxylamine to produce a volatile compound (a perfluorotriacetyl [PFTA] derivative) that was analyzed by negative chemical ionization-mass spectrometry. Selective ion monitoring was used on $m / z$ 435/436/437 (representing a loss of hydrofluoric acid from the molecular ion). Isotopic enrichment above natural occurrence was determined by subtraction of an unlabeled standard. The 2 derivatives gave identical results when enrichments were calculated with their respective standard curves. 
The fraction of labeled $\mathrm{CD} 5{ }^{+} \mathrm{CD} 19^{+}$cells, $f$, at each sampled time point was calculated as follows (26): $f=$ cellular $\%{ }^{2} \mathrm{H}$ enrichment / (equilibrium serum $\%{ }^{2} \mathrm{H}_{2} \mathrm{O}$ enrichment $\times A$ ), where $A$ is an empirically derived factor representing the degree of hydrogen atom exchange into deoxyribose during DNA replication. This value was determined to be 3.5 when the pentane-tetraacetate derivative was used and 3.07 for the PFTA derivative.

${ }^{2} \mathrm{H}_{2} \mathrm{O}$ equilibration correction. Total body ${ }^{2} \mathrm{H}_{2} \mathrm{O}$ does not immediately reach the stable level maintained during the maintenance phase of the experiment. More significantly, ${ }^{2} \mathrm{H}_{2} \mathrm{O}$ does not immediately return to zero during the washout phase. Modeling the body ${ }^{2} \mathrm{H}$ concentration as a function of time, $H(t)$, corrects for these delays. The 2 main physiologic parameters that affect the rate of equilibration and the equilibrium level are the patients' total body water, $W_{\text {body }}$, and the daily water turnover rate, $W_{\text {day }}$, including metabolic water. $W_{\text {body }}$ is estimated as 0.7 times the total body weight, as shown in Figure $1 \mathrm{~A}$. $W_{\text {day }}$ determines the equilibrium ${ }^{2} \mathrm{H}_{2} \mathrm{O}$ value, since at equilibrium the percentage ${ }^{2} \mathrm{H}_{2} \mathrm{O}$ enrichment, $\mathrm{H}_{\text {eq }}$, is equal to the daily incoming percentage ${ }^{2} \mathrm{H}_{2} \mathrm{O}$. Since our protocol calls for the ingestion of $60 \mathrm{ml}(0.061)$ of $70 \%{ }^{2} \mathrm{H}$ water per day, $H_{\mathrm{eq}}=$ $(0.06 \times 0.7) / W_{\text {day. }}$. Because $H_{\mathrm{eq}}$ was calculated for each subject from the percentage ${ }^{2} \mathrm{H}_{2} \mathrm{O}$ enrichment and the ${ }^{2} \mathrm{H}_{2} \mathrm{O}$ intake was known, $W_{\text {day }}$ was calculable. The fractional daily water exchange, $f_{\mathrm{w}}$, was $f_{\mathrm{w}}=W_{\text {day }} / W_{\text {body }}$. For patients whose body weight was not recorded $(n=6)$, a value of $6 \%$ was used (Supplemental Table 1).

For simplicity, a normalized body ${ }^{2} \mathrm{H}_{2} \mathrm{O}$ concentration, $h$, was defined as $h(t)=H(t) / H_{\text {eq }}$. The normalized percentage ${ }^{2} \mathrm{H}_{2} \mathrm{O}$ of the loading, $h_{\text {load, }}$, maintenance, $h_{\text {main }}$, and washout, $h_{\text {wash }}$, doses is then 3,1 , and 0 , respectively, since the loading dose is triple the maintenance dose. The latter established equilibrium. The function $h(t)$ was then modeled for each phase of the experiment using the differential equation for a well-mixed system, where the normalized incoming percentage ${ }^{2} \mathrm{H}_{2} \mathrm{O}, h_{\text {in }}$, is 1 of the 3 values above $h^{\prime}(t)=f_{\mathrm{w}}\left(h_{\mathrm{in}}-h(t)\right)$.

The initial condition for the loading phase is zero, since there is no label at time 0 and the initial conditions for the maintenance and washout phases are the solutions to the equation of the previous phase. A representative plot of $h(t)$ modeled from patients' parameters is shown in Figure 1D.

Single-compartment model. The single-compartment model used to analyze data (26) is diagrammed in Figure 2A. The fractional birth, $b$, and death, $d$, rates (in units of time ${ }^{-1}$ ) are assumed to be constant over the course of the study. The degree of cell labeling in newly born cells is equal to the percentage ${ }^{2} \mathrm{H}$ concentration, the normalized units of $h(t)$. Therefore, the rate of change in the number of labeled cells, $L(t)$, is described by the differential equation $L^{\prime}(t)=h(t) b v(t)-d L(t)$, where $v(t)$, the compartment size in number of cells, is defined by $v^{\prime}(t)=(b-d) v(t)$ to account for unequal birth and death rates. The fraction of labeled cells, $f(t)$, is then $L(t)$ divided by $v(t)$, which solves to $f(t)=h(t)+(f(0)-b(t)) e^{-b t}$.

It should be noted that the $d$ terms drop out and the function is therefore solely dependent on $b$. Because constant fractional birth and death rates are assumed, this equation is independent of any changes in compartment size but does not assume constant compartment size.

Two-compartment model. A 2-compartment mixture model was also developed, as diagrammed in Figure 2B. Since no data were obtained from the nonvascular compartment, a steady-state assumption was made to allow the equations to be solved. All absolute rates are therefore equal. Since $b$ is defined as the fractional birth rate of the first compartment, the exchange rate will also be $b$ and the death rate from the second compartment will be $b / v_{\mathrm{r}}$, where $v_{\mathrm{r}}$ is the relative sizes of the compartments defined as $v_{\mathrm{r}}=v_{2} / v_{1}$. The first compartment is described by the equations above such that $L_{1}{ }^{\prime}(t)=h(t) b v_{1}-b L_{1}(t)$ and $f_{1}(t)=e^{-b t}\left[f_{1}(0)+h(t)\left(e^{b t}-1\right)\right]$. The change in labeled cells in the second compartment is then $L_{2}^{\prime}(t)=b L_{1}(t)-b L_{2}(t) / v_{\mathrm{r}}$. The fraction of labeled cells in the second compartment, $f_{2}(t)$, is $L_{2}(t)$ divided by $v_{2}$, which solves to the following:

\section{Equation 1}

$$
f_{2}(t)=h(t)+f_{2}(0) e^{-b t v_{v_{r}}}+\frac{e^{-b t v_{r}}\left(f_{1}(0)-h(t)\right)}{v_{r}-1}+\frac{e^{-b t}\left(h(t)-f_{1}(0)\right)}{v_{r}-1}
$$

Since $b$ is a fraction of the first compartment, the total clonal birth rate, $B$, is $b /\left(1+v_{\mathrm{r}}\right)$. The SE of $B$ was calculated from the SEs of the first-compartment birth rate and relative compartment sizes obtained from the modelfitting software, using the following formula:

\section{Equation 2}

$$
S E_{B}=\frac{b}{1+v_{r}} \sqrt{\frac{S E_{b}^{2}}{b^{2}}+\frac{S E_{\left(1+v_{i}\right)}^{2}}{\left(1+v_{r}\right)^{2}}}
$$

Model fitting and statistical analyses. Models were fit to the data using the Levenberg-Marquardt method of nonlinear regression as implemented in OriginPro version 7-SR4 (OriginLab Corp.) and GraphPad Prism version 4.01 (GraphPad Software Inc.). Equations supplied to the curve-fitting algorithm were derived from the equations listed above using Mathematica version 5.0 (Wolfram Research Inc.) and are shown in Supplemental Figure 1 . The input values, modeled parameters, and statistical values of the parameters are shown in Supplemental Table 1.

\section{Acknowledgments}

We thank Scott Orenstein for technical assistance and Ann Way for patient coordination. These studies were supported in part by the NIH via RO1 grants from the National Cancer Institute (CA81554 and CA87956) and by a General Clinical Research Center Grant (M01 RR018535) from the National Center for Research Resources. The Peter J. Sharp Foundation, the Jean Walton Fund for Lymphoma and Myeloma Research, the Marks Family Foundation, the Joseph Eletto Leukemia Research Fund, the Tebil Foundation, the Horace W. Goldsmith Foundation Inc., and KineMed Inc., through an unrestricted gift, also provided support.

Received for publication September 20, 2004, and accepted in revised form December 20, 2004.

Address correspondence to: Nicholas Chiorazzi, Institute for Medical Research, North Shore-LIJ Health System, 350 Community Drive, Manhasset, New York 11030, USA. Phone: (516) 562-1001; Fax: (516) 562-1022; E-mail: nchizzi@nshs.edu.
1. Caligaris-Cappio, F., and Hamblin, T.J. 1999. B-cell
chronic lymphocytic leukemia: a bird of a different
feather. J. Clin. Oncol. 17:399-408.
2. Granziero, L., et al. 2001. Survivin is expressed on
CD40 stimulation and interfaces proliferation and
apoptosis in B-cell chronic lymphocytic leukemia.
Blood. 97:2777-2783.
3. Ghia, P., and Caligaris-Cappio, F. 2000. The indis- pensable role of microenvironment in the natural history of low-grade B-cell neoplasms. Adv. Cancer Res. 79:157-173.

4. Dighiero, G., et al. 1991. B-cell chronic lymphocytic leukemia: present status and future directions. French Cooperative Group on CLL. Blood. 78:1901-1914.

5. Dameshek, W. 1967. Chronic lymphocytic leukemia: an accumulative disease of immunolgically incompetent lymphocytes. Blood. 29(Suppl.):566-584.

6. Chu, P., et al. 2002. Latent sensitivity to Fasmediated apoptosis after CD40 ligation may explain activity of CD154 gene therapy in chronic lymphocytic leukemia. Proc. Natl. Acad. Sci. U. S. A. 99:3854-3859.

7. Marti, G.E., et al. 1992. Evolution of leukemic het- 
erogeneity of human B-CLL lymphocytes between and within patients. Curr. Top. Microbiol. Immunol. 182:303-311.

8. Rai, K.R., and Patel, D.V. 1995. Chronic lymphocytic leukemia. In Hematology: basic principles and practice. R. Hoffman, E. Benz, and S. Shattil, editors. Churchill Livingstone. New York, New York, USA. 1308-1321.

9. Damle, R.N., et al. 1999. Ig V gene mutation status and CD38 expression as novel prognostic indicators in chronic lymphocytic leukemia. Blood. 94:1840-1847.

10. Hamblin, T.J., Davis, Z., Gardiner, A., Oscier, D.G., and Stevenson, F.K. 1999. Unmutated $\operatorname{Ig} \mathrm{V}(\mathrm{H})$ genes are associated with a more aggressive form of chronic lymphocytic leukemia. Blood. 94:1848-1854.

11. Wiestner, A., et al. 2003. ZAP-70 expression identifies a chronic lymphocytic leukemia subtype with unmutated immunoglobulin genes, inferior clinical outcome, and distinct gene expression profile. Blood. 101:4944-4951.

12. Crespo, M., et al. 2003. ZAP-70 expression as a surrogate for immunoglobulin-variable-region mutations in chronic lymphocytic leukemia. N. Engl. J. Med. 348:1764-1775.

13. Jelinek, D.F., et al. 2001. Analysis of clonal B-cell CD38 and immunoglobulin variable region sequence status in relation to clinical outcome for B-chronic lymphocytic leukaemia. Br. J. Haematol. 115:854-861.

14. Krober, A., et al. 2002. V(H) mutation status, CD38 expression level, genomic aberrations, and survival in chronic lymphocytic leukemia. Blood. 100:1410-1416.

15. Hamblin, T.J., et al. 2002. CD38 expression and immunoglobulin variable region mutations are independent prognostic variables in chronic lymphocytic leukemia, but CD38 expression may vary during the course of the disease. Blood. 99:1023-1029.
16. Ghia, P., et al. 2003. The pattern of CD38 expression defines a distinct subset of chronic lymphocytic leukemia (CLL) patients at risk of disease progression. Blood. 101:1262-1269.

17. Dohner, H., et al. 2000. Genomic aberrations and survival in chronic lymphocytic leukemia. N. Engl. J. Med. 343:1910-1916

18. Montserrat, E., Sanchez-Bisono, J., Vinolas, N., and Rozman, C. 1986. Lymphocyte doubling time in chronic lymphocytic leukaemia: analysis of its prognostic significance. Br. J. Haematol. 62:567-575.

19. Molica, S., and Alberti, A. 1987. Prognostic value of the lymphocyte doubling time in chronic lymphocytic leukemia. Cancer. 60:2712-2716.

20. Simonsson, B., and Nilsson, K. 1980. ${ }^{3} \mathrm{H}$-thymidine uptake in chronic lymphocytic leukaemia cells. Scand. J. Haematol. 24:169-173.

21. Simonsson, B., Nilsson, K., Terenius, L., and Glimelius, B. 1978. Biochemical characteristics of chronic lymphocytic leukaemia. Glucocorticoid receptors, $B$ and $T$ lymphocyte surface markers, concanavalin A-induced agglutination and thymidine incorporation. Scand. J. Haematol. 21:379-389.

22. Stryckmans, P.A., Debusscher, L., and Collard, E. 1977. Cell kinetics in chronic lymphocytic leukaemia (CLL). Clin. Haematol. 6:159-167.

23. Zimmerman, T.S., Godwin, H.A., and Perry, S. 1968. Studies of leukocyte kinetics in chronic lymphocytic leukemia. Blood. 31:277-291.

24. Dormer, P., Theml, H., and Lau, B. 1983. Chronic lymphocytic leukemia: a proliferative or accumulative disorder? Leuk. Res. 7:1-10.

25. Schiffer, L.M., et al. 1969. Lymphocyte kinetics in chronic lymphocytic leukaemia (CLL) studied by ECIB. Br. J. Haematol. 17:408.

26. Neese, R.A., et al. 2002. Measurement in vivo of proliferation rates of slow turnover cells by $2 \mathrm{H} 2 \mathrm{O}$ labeling of the deoxyribose moiety of DNA. Proc Natl. Acad. Sci. U. S. A 99:15345-15350.

27. Cheson, B.D., et al. 1996. National Cancer Insti- tute-sponsored Working Group guidelines for chronic lymphocytic leukemia: revised guidelines for diagnosis and treatment. Blood. 87:4990-4997.

28. Schmid, C., and Isaacson, P.G. 1994. Proliferation centres in B-cell malignant lymphoma, lymphocytic (B-CLL): an immunophenotypic study. Histopathology. 24:445-451.

29. Pileri, S.A., et al. 2000. The pathologist's view point. I. Indolent lymphomas. Haematologica. 85:1291-1307.

30. Schiffer, L.M. 1968. Kinetics of chronic lymphocytic leukemia. Ser. Haematol. 1:3-23.

31. Themi, H., Trepel, F., Schick, P., Kaboth, W., and Begemann, H. 1973. Kinetics of lymphocytes in chronic lymphocytic leukemia: studies using continuous ${ }^{3} \mathrm{H}$-thymidine infusion in two patients. Blood. 42:623-636.

32. Plunkett, W., and Saunders, P.P. 1991. Metabolism and action of purine nucleoside analogs. Pharmacol. Ther. 49:239-268.

33. Manaster, J., Fruhling, J., and Stryckmans, P. 1973. Kinetics of lymphocytes in chronic lymphocytic leukemia. I. Equilibrium between blood and a "readily accessible pool." Blood. 41:425-438.

34. Bazerbashi, M.B., Reeve, J., and Chanarin, I. 1978. Studies in chronic lymphocytic leukaemia. The kinetics of 51Cr-labelled lymphocytes. Scand. J. Haematol. 20:37-51.

35. Chandra, P., et al. 1979. Correlation of total body potassium and leukemic cell mass in patients with chronic lymphocytic leukemia. Blood. 53:594-603.

36. Fais, F., et al. 1998. Chronic lymphocytic leukemia B cells express restricted sets of mutated and unmutated antigen receptors. J. Clin. Invest. 102:1515-1525.

37. Chen, L., et al. 2002. Expression of ZAP-70 is associated with increased B-cell receptor signaling in chronic lymphocytic leukemia. Blood. 100:4609-4614.

38. Macallan, D.C., et al. 1998. Measurement of cell proliferation by labeling of DNA with stable isotopelabeled glucose: studies in vitro, in animals, and in humans. Proc. Natl. Acad. Sci. U. S. A. 95:708-713. 